\title{
Association of adiponectin gene polymorphisms with hypertensive disorder complicating pregnancy and disorders of lipid metabolism
}

\author{
Y. Wang ${ }^{1}$, R.X. Liư ${ }^{2}$ and H. Liu' ${ }^{1}$ \\ ${ }^{1}$ Department of Obstetrics, Affiliated Hospital Weifang Medical University, \\ Weifang, China \\ 2Department of Obstetrics and Gynecology, Weifang Medical University Clinical School, \\ Weifang, China \\ Corresponding author: R.X. Liu \\ E-mail: wangying2015_y@163.com
}

Genet. Mol. Res. 14 (4): 15213-15223 (2015)

Received July 8, 2015

Accepted September 2, 2015

Published November 25, 2015

DOI http://dx.doi.org/10.4238/2015.November.25.9

ABSTRACT. The aim of this study was to determine whether single nucleotide polymorphisms (SNPs) in APM1 contribute to disorders of lipid metabolism in hypertensive disorder complicating pregnancy (HDCP). The study included 178 pregnant women with HDCP and 243 healthy pregnant controls. Using PCR-restriction fragment length polymorphism, we detected the frequencies of genotypes, alleles, and haplotypes of two SNPs, +45T>G (rs2241766) and +276G>T (rs1501299), in APM1. We found that the SNP +276 TT genotype was significantly associated with protection against HDCP compared to the pooled $\mathrm{G}$ genotypes. The genotype and allele frequency distributions of SNP +276 were significantly different between the cases and controls. Single-point genotype and allele distributions in SNP +45 were not statistically different between the groups. The pooled $G$ haplotypes were significantly overrepresented in the case group compared to the TT haplotype. Plasma adiponectin (APN) 
concentration was determined by enzyme-linked immunosorbent assay, and we found that APN levels in cases were significantly lower than those in controls. Using the clinical data, we evaluated the correlation between the two SNPs and HDCP development, and revealed an association between the two SNPs and disorders of lipid metabolism in patients with HDCP. Except for fasting insulin levels, which was higher in cases than in controls, there were no significant differences in the other clinical data between the two groups.

Key words: APM1; SNPs; HDCP; Disorders of lipid metabolism

\section{INTRODUCTION}

Hypertensive disorder complicating pregnancy (HDCP) is a common but severe complication, with symptoms that include transient hypertension (systolic blood pressure: SBP $\geq 140 \mathrm{mmHg}$ and/or diastolic blood pressure: DBP $\geq 90 \mathrm{mmHg}$ ), edema, and proteinuria, that occurs after the 20th week of pregnancy through the 2nd week postpartum, but often disappears after delivery. According to Williams Obstetrics, HDCP, which includes hypertension of pregnancy, preeclampsia (mild and severe), eclampsia, chronic hypertension with preeclampsia, and pregnancy with chronic hypertension affected 7 to $12 \%$ of pregnancies in other countries and $9.4 \%$ of pregnancies in China (Cunningham et al., 2014). HDCP greatly influences mother and infant health. For example, HDCP in pregnant women may lead to convulsions, coma, heart and renal failure, or even death. In infants, placental insufficiency and hypofunction induced by uterine vasospasm can lead to infant distress, retardation, fetal death, and neonatal death (Yue, 2008). Furthermore, HDCP is one of the reasons for the increasing prevalence of maternal and perinatal mortality. Preeclampsia is characterized by the symptoms of HDCP with accompanying headache, dizziness, blurred version, epigastric discomfort, nausea, etc., before convulsions, which can precede eclampsia.

For a century, many theories have tried to explain the mechanism underlying HDCP, which have included dysfunction of the placenta, endothelium dysfunction, disorders of lipid metabolism, heredity, and inflammatory reactions. However, the etiology and pathogenesis have remained unclear, which has created difficulties for the prevention and treatment of HDCP. Recently, with the exception of low-age or elderly primiparity, high-risk factors for HDCP development including nutritional deficiency, multiple pregnancy with a history of essential hypertension (EH), nephritis, and diabetes mellitus have been shown to be genetically influenced by the interplay of multiple genes or polymorphic sites (Harrison et al., 1997; Serrano et al., 2004). Since the 19th century, familial preeclampsia has been confirmed, which shows that genetic factors play an important role in the development of HDCP (Kruglyak, 1999). Currently, the detection of HDCP mainly depends on clinical symptoms combined with biochemical indices, but these diagnostic methods do not provide early warnings. As the development and clinical application of biological techniques in prenatal diagnosis increases, especially the development of those in molecular biology and medical genetics, more and more diseases could be safely and accurately diagnosed during early embryo development.

Common genetic variation, such as single nucleotide polymorphisms (SNPs) of key genes or haplotype combinations, could be important in the susceptibility of complex diseases. As a type 
of base substitution, SNPs are a common biallelic variation caused by transition or transversion of a single nucleotide with a frequency $>1 \%$. SNPs in the coding and regulatory regions may change gene expression levels or the function of the gene products, and have thus become the most commonly studied genetic polymorphisms in the fields of genetics, human genomics, and pharmacogenomics (Riley et al., 2000; John et al., 2004). As such, the development of biological techniques has made the estimation of allelic frequency of SNPs easier. Technically, there has been a massive implementation of automatic analysis of biallelic SNPs. Computer analysis has therefore made it easy to study the correlation between SNPs and the susceptibility to complex diseases. As techniques have continued to advance, methods for simple and non-invasive detection of SNPs have garnered more and more attention. Therefore, the identification of genes or polymorphic sites that increase the susceptibility of HDCP will help confirm the genetic basis of HDCP development. This in turn may lead to the prevention, early diagnosis, and treatment of HDCP, which could be accomplished through a combination of molecular biological and clinical approaches.

Adiponectin (APN) is a specific type of protein hormone secreted by adipocytes that plays an important role in regulating lipid metabolism, sugar metabolism, and insulin resistance, and has anti-atherosclerosis and anti-inflammatory activities (Maeda et al., 2002; Yamauchi et al., 2002). The mRNA transcript that encodes for APN is the most abundant gene transcript in adipose tissue, and thus was named Adipose most abundant gene transcript 1 (APM1). The APM1 gene is located on chromosome $3 q 27$. The full-length of $A P M 1$ is $17 \mathrm{~kb}$ with 3 exons and 2 introns, and contains multiple SNPs. The coding region starts from the second exon and ends at the front of the third exons, encoding for a total of 244 amino acids with a $30-\mathrm{kDa}$ molecular weight, and is found at the susceptibility loci for metabolic syndrome (Kissebah et al., 2000; Matsuda et al., 2002). Much research has concentrated on the relationship between APN and HDCP with idea that disorders of lipid metabolism could lead to vascular endothelial cell injury, which could in turn be related to metabolic hypertension, cardiovascular diseases, type 2 diabetes mellitus, and HDCP among other disorders (Francke et al., 2001; Ramsay et al., 2003).

Many researchers have shown that APN is correlated with lipid metabolism. For example, Lindsay et al. (2002) demonstrated that there was a significant negative correlation between APN and triglycerides (TG). Wolf et al. (2004) showed that while insulin resistance played a role in the development of preeclampsia, APN had an effect on the regulation of insulin and sugar metabolism, and had anti-inflammation and anti-atherosclerosis activity; therefore relieving vascular endothelial injury. D'Anna et al. (2005) measured the plasma APN concentration of 1842 pregnant women in the first trimester and followed their pregnancy outcomes. The authors found that the plasma APN concentration in the gestational hypertension group during the second and third trimesters was significantly lower than that in the control group, and further found that the APN concentration in the preeclampsia group was significantly lower than that in the gestational hypertension group. Moreover, there was a negative correlation between the plasma APN concentration and maternal age, gestational age, maternal body mass index, SBP, and content of urinary protein in the preeclampsia group, indicating that hypoadiponectinemia was significantly correlated to an increased risk of gestational hypertension and preeclampsia. Therefore, we hypothesized that APN plays a role in the underlying mechanism of preeclampsia development. Regarding SNPs of APM1, Zhang et al. (2011) studied 5 SNPs in the APM1 promoter region and found that 1) $-11426 A>G$ and -11156 ins $C / A$ were in complete linkage disequilibrium (LD, $D^{\prime}=1 ; r^{2}=1$ ), indicating that $-11426 \mathrm{G}$ could be the TagSNP of $\mathrm{EH}$ risk haplotype without measuring $-11156 \mathrm{insCA} ; 2$ ) 
-11426G and -11426G-11377C were EH risk factors and EH risk haplotypes in the Han of Yanbian region; 3) in the EH group, the plasma APN level was significantly lower than that in the control group. Furthermore, the study of Avery et al. (2011) showed that the -11377C>G (rs266729) SNP was an independent factor influencing SBP of patients with hypertension. Wang et al. (2009) evaluated the correlation between type 2 diabetes mellitus (T2DM) and APM1 SNPs, including those in the second intron (IVS2) $+712 A>G$ ( $r$ 3774261) and +349A>G ( $r 2241767)$; third exon (Exon3) $+331 \mathrm{~T}>\mathrm{C}$ (rs17366743); promoter region -11391G>A and -11377C>G; and in the 3'-UTR $+4545 \mathrm{G}>\mathrm{C}$ ( $\mathrm{rs} 1063539)$. The authors found that in the IVS2 +712A > G SNP, there was a correlation between the GG genotype and T2DM, in which plasma TC and LDL levels were increased. The polymorphism of $A \rightarrow G$ increased the risk of T2DM and metabolism disorder of blood lipid. The authors further demonstrated that the polymorphisms of $-11377 \mathrm{C}>\mathrm{G}$ and $+4545 \mathrm{G}>\mathrm{C}$ were correlated to obese and non-obese T2DM separately, indicating that they were both susceptible genotypes of obese and non-obese T2DM. In comparing preeclamptic patients to the control subjects, Saarela et al. (2006) found that in the second intron region +276T>G of APM1, the TT genotype was a protective factor against preeclampsia. Furthermore, there was a significant difference between the allelic frequency of the two groups at site $+276 \mathrm{~T}>\mathrm{G}$, while there was no significant difference at site $+45 T>G$. Tang et al. (2008) demonstrated that both the polymorphism of $A P M 1+45 T>G$ and the plasma APN concentration were correlated to hypertension. While studying patients with severe preeclampsia, Bai et al. (2010) found that APN concentration, SBP, and 24-h proteinuria levels of patients with the TT genotype at site $+45 \mathrm{~T}>\mathrm{G}$ were significantly lower than those in $\mathrm{G}$ allele carriers ( $T G+G G)$, which identified APN as a new susceptibility gene for $\mathrm{EH}$.

Most recently, research regarding APM1 and HDCP has only concentrated on particular regions or sites, with less attention being spent on the combination effect of multiple regions and multiple sites of APM1, such as correlation analyses between haplotypes and diseases or between haplotypes and clinical and/or biochemical indices. In China, there has been limited reporting on the correlations between APM1 SNP genotypes, alleles, or haplotype combinations and disorders of lipid metabolism, such as HDL-C, TG, and ApoB in patients with gestational hypertension. Whether the correlations mentioned above could lead to vascular endothelial injury, which could influence HDCP development remains unclear. Therefore, confirmation of the correlation between disorders of lipid metabolism and vascular endothelial injury may lead to the prevention, prediction, and treatment of HDCP, which may also play an important role in enhancing the health of mother and infant while decreasing maternal and perinatal mortality.

\section{MATERIAL AND METHODS}

\section{Subjects}

Written approval for the study was obtained from the Zhongshan Hospital Xiamen University. Informed consent was obtained from all patients and controls. Information was collected retrospectively in connection with 178 single pregnant women with HDCP, average age: $27.52 \pm 6.33$ years, gestational age: $38.10 \pm 2.86$ weeks, and without history of chronic hypertension, nephritis, diabetes mellitus, or cardiovascular disease. The control group was comprised of 243 healthy pregnant women with no history of HDCP, average age: $28.65 \pm 5.69$ years, and gestational age: $37.81 \pm 3.09$ weeks. All participants were recruited from the Han of Fujian Province at Zhongshan Hospital between August 2005 to August 2007, and had no genetic relationship. All diagnostic criteria were referenced to the Obstetrics and Gynecology (Yue, 2008). 


\section{Genomic DNA extraction}

Blood was drawn from the antecubital vein into EDTA tubes and samples were stored at $-80^{\circ} \mathrm{C}$. DNA was extracted by means of the TGuide blood genomic DNA extraction kit (OSR-M102), obtained from TIANGENG Biotech (Beijing) Co., Ltd. following manufacturer instructions.

\section{DNA concentration and purification detection}

The ratio of the absorbance at 260 and $280 \mathrm{~nm}$ of total DNA measured with an ultraviolet spectrophotometer was used to assess DNA purity, and the content of total DNA was calculated. DNA was stored at $-80^{\circ} \mathrm{C}$ prior to use.

\section{PCR amplification primers}

The primers for PCR amplification were designed with the Primer Premier5 software and reference literature by Shanghai Generay Biotech Co., Ltd., with specification $10 D^{*} 2$. Dry powder primers were centrifuged at 12,000 rpm for $2 \mathrm{~min}$, dissolved in Tris-EDTA buffer solution (10 mM Tris$\mathrm{HCl}, 1 \mathrm{mM}$ EDTA, pH 8.0), kept as $100 \mu \mathrm{M}$ mother liquor, separated, and stocks were stored at $-20^{\circ} \mathrm{C}$. Stocks thawed and diluted as needed. The primers and restriction enzyme sites for APM1 SNPs are shown in Table 1. Amplification conditions and system are shown in Tables 2 and 3.

Table 1. Primer sequences, annealing temperatures, lengths, and restriction enzymes.

\begin{tabular}{llccr}
\hline Site & Primer sequence & Annealing temperature $\left({ }^{\circ} \mathrm{C}\right)$ & Length (bp) & Restriction endonuclease \\
\hline$+45 T>G($ rs2241766) & F: 5'-CTGAGATGGACGGAGTCCTTT-3' & $60^{\circ}$ & 456 & Smal \\
& R: 5'-CCAAATCACTTCAGGTTGCTT-3' & & \\
$+276 G>T$ (rs1501299) & F: 5'-CTGAGATGGACGGAGTCCTTT-3' & $59^{\circ}$ & 456 & Bsml \\
& R: 5'-CCAAATCACTTCAGGTTGCTT-3' & & & \\
\hline
\end{tabular}

F: forward primer; R: reverse primer.

\begin{tabular}{|c|c|c|c|}
\hline Step & Temperature $\left({ }^{\circ} \mathrm{C}\right)$ & Time & Cycle number \\
\hline Predegeneration & $94^{\circ}$ & $5 \mathrm{~min}$ & \\
\hline Degeneration & $94^{\circ}$ & $30 \mathrm{~s}$ & 30 cycles \\
\hline Annealing & $59^{\circ}-61^{\circ}$ & $30 \mathrm{~s}$ & \\
\hline Extension & $72^{\circ}$ & $30 \mathrm{~s}$ & \\
\hline Extension terminal & $72^{\circ}$ & $7 \mathrm{~min}$ & 1 cycle \\
\hline Storage & $4^{\circ}$ & $\rightarrow \infty$ & \\
\hline
\end{tabular}

Table 3. PCR amplification system (20- $\mu \mathrm{L}$ reaction system).

\begin{tabular}{lc}
\hline Composition & Volume $(\mu \mathrm{L})$ \\
\hline Ultrapure water & 7 \\
2X PCR Mix & 10 \\
Primer & 1 \\
F & 1 \\
R & 1 \\
Template DNA & 20 \\
Total volume & 20 \\
\hline
\end{tabular}




\section{Genotype identification by RFLP ( $30-\mu L$ reaction system)}

Reactions were incubated overnight at $37^{\circ} \mathrm{C}$. The PCR products were then separated and purified by $3 \%$ agarose gel electrophoresis, and stained with ethidium bromide. The genotypes were identified with Epichemi 3 Darkroom image acquisition and analysis system from UVP Co., Ltd. Reaction components are provided in Table 4.

\begin{tabular}{lc}
\multicolumn{1}{c}{ Table 4. Genotype identification by restriction fragment length polymorphism $(30-\mu L$ reaction system). } \\
\hline Composition & Volume $(\mu \mathrm{L})$ \\
\hline Ultrapure water & 17 \\
PCR products & 10 \\
10X Green Buffer & 2 \\
Restriction endonuclease & 1 \\
Total volume & 30 \\
\hline
\end{tabular}

\section{Plasma APN concentration measurement by enzyme-linked immunosorbent assay (ELISA)}

Blood samples were taken after an overnight fast. Time of sampling was standardized at 8-9 am. The samples were venous blood, and tripotassium ethylenediamine tetraacetic acid anticoagulant was added. The samples were stored at $-80^{\circ} \mathrm{C}$ before use. Plasma APN was evaluated in the blood samples of the cases and the controls as measured by sandwich ELISA with a commercially available APN ELISA kit (Linco Research Inc., St. Charles, MO, USA).

\section{Statistical analysis}

The polymorphism allele frequencies were consistent with Hardy-Weinberg equilibrium. LD was analyzed by the SHEsis online analysis software. Means, proportions, and SDs ( \pm ) were used for descriptive purposes. Data are reported as means \pm SD. Correlations between parameters were examined by means of Pearson (a value of $P>0.05$ ) correlation coefficients, and using logtransformed parameters where required. A value of $P<0.01$ was considered to be statistically significant.

\section{RESULTS}

\section{Clinical data}

Patient characteristics and clinical data for the cases and controls are shown in Table 5.

\section{Correlations between the influencing factors and HDCP}

Demographic characteristics of the cases and control subjects are shown in Table 2. There were no significant differences in BMI, SBP, DBP, LDL-C, and HDL-C between the cases and control subjects. Gestational age at delivery was earlier in women with HDCP, but this difference did not reach significance compared to that in control subjects. Fasting insulin was significantly higher in women with HDCP than that in the control group $(P>0.01)$. 
Table 5. Clinical characteristic of women with (case group) or without (control group) HDCP (means \pm SD).

\begin{tabular}{lccc}
\hline Variable & Case $(\mathrm{N}=178)$ & Control $(\mathrm{N}=243)$ & $\mathrm{P}$ \\
\hline Age (years) & $27.52 \pm 6.33$ & $28.65 \pm 5.69$ & 0.899 \\
Gestational age at delivery (weeks) & $35.10 \pm 2.86$ & $37.81 \pm 3.09$ & $<0.01$ \\
BMI $\left(\mathrm{kg} / \mathrm{m}^{2}\right)$ & $28.88 \pm 5.01$ & $23.97 \pm 2.80$ & $<0.01$ \\
SBP $(\mathrm{mmHg})$ & $153.79 \pm 19.77$ & $131.56 \pm 14.13$ & $<0.01$ \\
DBP (mmHg) & $104.33 \pm 15.32$ & $90.87 \pm 7.90$ & $<0.01$ \\
Fasting insulin (mIU/L) & $22.35 \pm 12.75$ & $13.47 \pm 7.78$ & 0.093 \\
LDL-C (mM) & $2.01 \pm 0.49$ & $1.90 \pm 0.33$ & $<0.01$ \\
HDL-C (mM) & $1.09 \pm 0.29$ & $1.35 \pm 0.20$ & $<0.01$ \\
\hline
\end{tabular}

$\mathrm{BMI}=$ body mass index; $\mathrm{SBP}=$ systolic blood pressure; $\mathrm{DBP}=$ diastolic blood pressure.

\section{Allele frequencies and distribution}

In SNP +276, the T allele was significantly protective against HDCP ( $<<0.05$; Pearson). Additionally, the genotype distribution of SNP +276 differed between the cases and controls $(P$ $<0.05$; Pearson) where the TT genotype was associated with protection against the condition compared to the $G G$ and $G T$ genotype $(P<0.05$; Pearson). The allele and genotype frequencies of SNP +45 did not significantly differ between the cases and controls $(P>0.05$; Pearson; Table 6$)$.

\begin{tabular}{|c|c|c|c|c|c|c|c|}
\hline \multirow[t]{2}{*}{ Genotype } & \multirow[t]{2}{*}{ Group } & \multirow[t]{2}{*}{ Number of samples } & \multicolumn{3}{|c|}{ Genotype (\%) } & \multicolumn{2}{|c|}{ Allele (\%) } \\
\hline & & & $\mathrm{T} / \mathrm{T}$ & $T / G$ & $\mathrm{G} / \mathrm{G}$ & $T$ & G \\
\hline \multirow[t]{2}{*}{ SNP +45 (exon 2) } & Case $(N=178)$ & 178 & $156(87.6)$ & $22(12.4)$ & $0(0)$ & $334(93.8)$ & $22(6.2)$ \\
\hline & Control $(N=243)$ & 243 & $220(91.0)$ & $22(9.1)$ & $1(0.4)$ & $462(95.1)$ & $24(4.9)$ \\
\hline \multirow[t]{2}{*}{ SNP +276 (intron 2) } & Case $(N=178)$ & 178 & $5(2.8)$ & $80(44.9)$ & $93(52.2)$ & $90(25.3)$ & $266(74.8)$ \\
\hline & Control $(N=243)$ & 190 & $25(10.3)$ & 109 (44.9) & $109(44.9)$ & $159(32.7)$ & 327 (67.3) \\
\hline
\end{tabular}

Asymptotic $P$ values for the SNP +45 (exon 2) genotype were $P=0.489$ and $P=0.510$ for cases and controls, respectively. Pooled SNP +45 genotype frequencies (GG vs GT and TT) were not significantly different between the cases and controls.

Asymptotic $P$ values for the SNP +276 (intron 2) genotype were $P=0.033$ and $P=0.043$ for cases and controls, respectively. Pooled SNP +276 genotype frequencies (TT vs TG and GG) were significantly different between the cases and controls $(P=0.012)$.

Both SNP +45 and SNP +276 were found to be in Hardy-Weinberg equilibrium, in the cases and controls.

\section{APN concentration}

The plasma APN concentrations were determined via ELISA. APN levels in cases were $8.87 \pm 6.01 \mu \mathrm{g} / \mathrm{mL}$, while in controls the levels were $12.09 \pm 3.33 \mu \mathrm{g} / \mathrm{mL}$. The APN concentration in cases was significantly lower than that in the control group $(P>0.01)$.

\section{Haplotype}

We used the genotype data from the exon 2 SNP and the intron 2 SNP in cases and controls to carry out a haplotype estimation analysis (Table 7). Estimated overall pairs of locus 
haplotype frequencies were not significantly different between the two groups $(P>0.05)$, whereas in the single-haplotype association analysis, the TT haplotype was significantly less common in cases than in controls $(P<0.05)$.

$\begin{aligned} & \text { Table 7. Estimated haplotype frequency distributions of the APN gene polymorphisms (SNP }+45 \text { and SNP }+276) \text { on } \\
& \text { chromosomes from women with (case group) }\end{aligned}$
\begin{tabular}{lcc}
\hline Haplotype & & Hithout (control group) HDCP. \\
\cline { 2 - 3 } & Case $(\mathrm{N}=356)$ & Control $(\mathrm{N}=486)$ \\
\hline T-G & $0.728 \pm 0.035$ & $0.656 \pm 0.029$ \\
T-T & $0.237 \pm 0.026$ & $0.311 \pm 0.024$ \\
G-G & $0.035 \pm 0.015$ & $0.033 \pm 0.008$ \\
\hline
\end{tabular}

Pooled haplotype frequencies (T-T vs pooled T-G and G-G) differed with statistical significance $(P<0.05)$ between the cases and controls.

\section{DISCUSSION}

Recently, HDCP has become one of the most important conditions leading to increased mortality of pregnant women and perinatal infants. Compared to EH, HDCP has a more complicated mechanism, and is causally associated with proteinuria during pregnancy. In addition to known HDCP risk factors that include nutritional deficiency, multiple pregnancies, a history of $\mathrm{EH}$, nephritis, and diabetes mellitus, HDCP has been shown to have heritable factors influenced by the interplay of multiple genes or multiple SNPS, with the exception of low-age or elderly primipara (Harrison et al., 1997; Serrano et al., 2004). Studies on patients from different families have also confirmed this heritable link (O'Shaughnessy et al., 2000; Chappell and Morgan, 2006). Because of the onset of symptoms during pregnancy, it is more important to know how the hereditary factors play a role in disease development compared to other hereditary diseases. HDCP studies should focus on the synergistic effects of multiple genes and SNPs because single SNPs may only slightly influence disease development, while the combined effect of multiple relative genes may have a profound effect on the disease. Previous studies have shown that disorders of lipid metabolism and hypoadiponectinemia were related to abnormal reactions of endothelium-dependent vasodilation in HDCP patients (Shimabukuro et al., 2003; Ouchi et al., 2003). APN is a plasma protein/hormone secreted by adipocytes, and is one of the most abundant proteins expressed in adipose tissues (Furukawa et al., 2004). Prior studies have shown that APN was the only down-regulated hormone of all adipocyte cytokines, which played a crucial role in glucose and lipid metabolism of patients with insulin resistance or cardiovascular diseases (Bacci et al., 2004; Nedvidkova et al., 2005). The development of molecular biology techniques has enhanced studies into the underlying genes that increase susceptibility of HDCP. Through genome-wide association studies, scientists have revealed that chromosomal region $3 q 27$ contained the susceptibility gene for metabolic syndrome, which encoded for APM1 (Guo et al., 2006). Therefore, scientists speculated that APM1 could affect metabolism by regulating APN levels, which may also play an unknown role in lipid metabolism disorder (Maeda et al., 2002; Yamauchi et al., 2002). Hyperlipidemia can influence blood vessel endothelial injury, while HDCP has been shown to be closely correlated to both endothelial injury and functional changes (Germain et al., 2007; Gilbert et al., 2008). Therefore, through molecular biological techniques, we studied the relationship between APM1 SNPs and lipid metabolism, and expected to find a genetic link between disorders of lipid metabolism and HDCP as this would offer a new way of thinking about HDCP etiology. 
APN, as an adipose tissue-derived protein, has been shown to have a protective role in insulin resistance, inflammation, endothelial dysfunction, obesity, and dyslipidemia (Redman et al., 1999; Lindsay et al., 2002; Engeli et al., 2003; Spranger et al., 2003). Moreover, all of these disorders have been connected with HDCP in the literature (Redman et al., 1999). Polymorphisms within the APN gene have shown marked correlations with different features of the metabolic syndrome (Filippi et al., 2004). Here, we explored the possible correlations between two SNPs in APM1 and HDCP. The results herein indicate a correlation between SNP +276 and HDCP as determined by the single-point association analysis. The minor TT genotype was significantly protective against HDCP, especially when compared with the pooled G genotypes. SNP +45 was not associated with HDCP risk in the allele and genotype frequency analysis. According to the haplotype estimation analysis, the TT haplotype was less frequent in the case group compared to the GG and GT haplotypes.

SNP +45 and SNP +276 were not likely to be functional polymorphisms because SNP +45 was a silent polymorphism and SNP + 276 was located in an intron. However, due to the association of SNP +276 with HDCP, it is possible that SNP +276 was in LD with a functional variant located elsewhere in the gene. The association of SNP +276 with T2DM had been linked to the genotype GG or the G allele (Hara et al., 2002), and with obesity and insulin resistance to the T allele (Filippi et al., 2004). In the current study, the decrease in HDCP risk involved the T allele. In other populations, it has been shown that there was an association between SNP +45 and T2DM, obesity, and insulin resistance (Stumvoll et al., 2002; Hara et al., 2002), although no significant correlation between HDCP and SNP +45 was observed herein. However, there could be differences in the LD structure across populations, leading to different risk alleles in the same SNPs.

The magnitude of the increase of plasma APN levels in cases above those in controls suggests that free-fatty acids and TG could help to predict HDCP when measured early in pregnancy or even prior to pregnancy. Additionally, it has advantages over lipid measurements as plasma APN levels have been shown to be unaltered in the postprandial state (Peake et al., 2003). D' Anna et al. (2005) found that plasma APN levels in patients with preeclampsia were lower than those in healthy pregnant women. Ramsay et al. (2003) also found that plasma APN levels were significantly higher in preeclamptic patients compared to those in healthy controls, although the APN levels were unrelated to APN mRNA expression in the placenta. These results suggested that the elevation of APN may have been a physiological response to avoid damage to endothelial cells. Moreover, the extent of the increase in APN levels suggests that it may be a particularly sensitive predictor of HDCP, but this will require further examination.

The key finding of this study was that there was a significant decrease in the risk of HDCP in carriers of the minor T allele and the TT genotype of SNP +276. The biological relevance of this finding requires further investigation with larger sample sizes. For example, the association of the less frequent PPARy Pro12Ala polymorphism with decreased risk of T2DM could not be confirmed until a meta-analysis was performed by Altshuler et al. (2000). In a similar manner, large population samples are likely necessary to show the associations linked to polygenic diseases. In conclusion, the results of the present study indicate that the APN gene is a promising candidate susceptibility gene for HDCP. Further investigations are needed to replicate our findings in other populations.

\section{Conflicts of interest}

The authors declare no conflict of interest. 


\section{ACKNOWLEDGMENTS}

Research supported by the Department of Obstetrics and Gynecology of Weifang Medical University, Weifang City, China.

\section{REFERENCES}

Altshuler D, Hirschhorn JN, Klannemark M, Lindgren CM, et al. (2000). The common PPARgamma Pro12Ala polymorphism is associated with decreased risk of type 2 diabetes. Nat. Genet. 26: 76-80.

Avery PJ, Patel SK, Ibrahim IM, Walker M, et al. (2011). Common variation in the adiponectin gene has an effect on systolic blood pressure. J. Hum. Hypertens. 25: 719-724.

Bacci S, Menzaghi C, Ercolino T, Ma X, et al. (2004). The $+276 \mathrm{G} / \mathrm{T}$ single nucleotide polymorphism of the adiponectin gene is associated with coronary artery disease in type 2 diabetic patients. Diabetes Care 27: 2015-2020.

Bai YP, Xiong W, Lin W, Jia J, et al. (2010). Relationships among adiponectin gene polymorphisms, proteinuria and increased blood pressure in the context of placental diseases. Hypertens. Res. 33: 1066-1070.

Chappell S and Morgan L (2006). Searching for genetic clues to the causes of pre-eclampsia. Clin. Sci. 110: 443-458.

Cunningham F, Leveno K, Bloom S, Spong CY, et al. (2014). Williams Obstetrics 24/E. McGraw Hill Professional, New York.

D'Anna R, Baviera G, Corrado F, Giordano D, et al. (2005). Plasma adiponectin concentration in early pregnancy and subsequent risk of hypertensive disorders. Obstet. Gynecol. 106: 340-344.

Engeli S, Feldpausch M, Gorzelniak K, Hartwig F, et al. (2003). Association between adiponectin and mediators of inflammation in obese women. Diabetes 52: 942-947.

Filippi E, Sentinelli F, Trischitta V, Romeo S, et al. (2004). Association of the human adiponectin gene and insulin resistance. Eur. J. Hum. Genet. 12: 199-205.

Francke S, Manraj M, Lacquemant C, Lecoeur C, et al. (2001). A genome-wide scan for coronary heart disease suggests in Indo-Mauritians a susceptibility locus on chromosome $16 \mathrm{p} 13$ and replicates linkage with the metabolic syndrome on 3q27. Hum. Mol. Genet. 10: 275127-275165.

Furukawa K, Hori M, Ouchi N, Kihara S, et al. (2004). Adiponectin down-regulates acyl-coenzyme A:cholesterol acyltransferase-1 in cultured human monocyte-derived macrophages. Biochem. Biophys. Res. Commun. 317: 831-836.

Germain AM, Romanik MC, Guerra I, Solari S, et al. (2007). Endothelial dysfunction: a link among preeclampsia, recurrent pregnancy loss, and future cardiovascular events? Hypertension 49: 90-95.

Gilbert JS, Ryan MJ, LaMarca BB, Sedeek M, et al. (2008). Pathophysiology of hypertension during preeclampsia: linking placental ischemia with endothelial dysfunction. Am. J. Physiol. Heart Circ. Physiol. 294: H541-H550.

Guo X, Saad MF, Langefeld CD, Williams AH, et al. (2006). Genome-wide linkage of plasma adiponectin reveals a major locus on chromosome 3q distinct from the adiponectin structural gene: the IRAS family study. Diabetes 55: 1723-1730.

Hara K, Boutin P, Mori Y, Tobe K, et al. (2002). Genetic variation in the gene enconding adiponection is associated with an increased risk of type 2 diabetes in the Japanese population. Diabetes 51: 536-540.

Harrison GA, Humphrey KE, Jones N, Badenhop R, et al. (1997). A genomewide linkage study of preeclampsia/eclampsia reveals evidence for a candidate region on 4q. Am. J. Hum. Genet. 60: 1158-1167.

John S, Shephard N, Liu G, Zeggini E, et al. (2004). Whole-genome scan, in a complex disease, using 11,245 single-nucleotide polymorphisms: comparison with microsatellites. Am. J. Hum. Genet. 75: 54-64.

Kissebah AH, Sonnenberg GE, Myklebust J, Goldstein M, et al. (2000). Quantitative trait loci on chromosomes 3 and 17 influence phenotypes of the metabolic syndrome. Proc. Natt. Acad. Sci. U. S. A. 97: 14478-14483.

Kruglyak $L$ (1999). Prospects for whole-genome linkage disequilibrium mapping of common disease genes. Nat. Genet. 22: 139-144.

Lindsay RS, Funahashi T, Hanson RL, Matsuzawa Y, et al. (2002). Adiponectin and development of type 2 diabetes in the Pima Indian population. Lancet 360: 57-58.

Maeda N, Shimomura I, Kishida K, Nishizawa H, et al. (2002). Diet-induced insulin resistance in mice lacking adiponectin/ ACRP30. Nat. Med. 8: 731-737.

Matsuda M, Shimomura I, Sata M, Arita Y, et al. (2002). Role of adiponectin in preventing vascular stenosis. The missing link of adipo-vascular axis. J. Biol. Chem. 277: 37487-37491.

Nedvidkova J, Smitka K, Kopsky V and Hainer V (2005). Adiponectin, an adipocyte-derived protein. Physiol. Res. 54: 133-140.

Ouchi N, Ohishi M, Kihara S, Funahashi T, et al. (2003). Association of hypoadiponectinemia with impaired vasoreactivity. Hypertension 42: 231-234.

O'shaughnessy KM, Ferraro F, Fu B, Downing S, et al. (2000). Identification of monozygotic twins that are concordant for 
preeclampsia. Am. J. Obstet. Gynecol. 182: 1156-1157.

Peake PW, Kriketos AD, Denyer GS, Campbell LV, et al. (2003). The postprandial response of adiponectin to a high-fat meal in normal and insulin-resistant subjects. Int. J. Obes. Relat. Metab. Disord. 27: 657-662.

Ramsay JE, Jamieson N, Greer IA and Sattar N (2003). Paradoxical elevation in adiponectin concentrations in women with preeclampsia. Hypertension 42: 891-894.

Redman CW, Sacks GP and Sargent IL (1999). Preeclampsia: an excessive maternal inflammatory response to pregnancy. Am. J. Obstet. Gynecol. 180: 499-506.

Riley JH, Allan CJ, Lai E and Roses A (2000). The use of single nucleotide polymorphisms in the isolation of common disease genes. Pharmacogenomics 1: 39-47.

Saarela T, Hiltunen M, Helisalmi S, Heinonen S, et al. (2006). Adiponectin gene haplotype is associated with preeclampsia. Genet. Test. 10: 35-39.

Serrano NC, Casas JP, Díaz LA, Páez C, et al. (2004). Endothelial NO synthase genotype and risk of preeclampsia: a multicenter case-control study. Hypertension 44: 702-707.

Shimabukuro M, Higa N, Asahi T, Oshiro Y, et al. (2003). Hypoadiponectinemia is closely linked to endothelial dysfunction in man. J. Clin. Endocrinol. Metab. 88: 3236-3240.

Spranger J, Kroke A, Mohlig M, Bergmann MM, et al. (2003). Adiponectin and protection against type 2 diabetes mellitus. Lancet 361: 226-228.

Stumvoll M, Tschritter O, Fritsche A, Staiger H, et al. (2002). Association of the T-G polymorphism in adiponectin (exon 2) with obesity and insulin sensitivity: interaction with family history of type 2 diabetes. Diabetes 51: 37-41.

Tang XM and Li NJQ (2008). Progress adiponectin gene polymorphism with essential hypertension. Yixueyuzhexue 29: 58-59.

Wang X, Zhang S, Chen Y, Liu H, et al. (2009). APM1 gene variants -11377C/G and 4545G/C are associated respectively with obesity and with non-obesity in Chinese type 2 diabetes. Diabetes Res. Clin. Pract. 84: 205-210.

Wolf M, Hubel CA, Lam C, Sampson M, et al. (2004). Preeclampsia and future cardiovascular disease: potential role of altered angiogenesis and insulin resistance. J. Clin. Endocrinol. Metab. 89: 6239-6243.

Yamauchi T, Kamon J, Minokoshi Y, Ito Y, et al. (2002). Adiponectin stimulates glucose utilization and fatty-acid oxidation by activating AMP-activated protein kinase. Nat. Med. 8: 1288-1295.

Yue J (2008). Preterm. Obstetrics and Gynecology. 7th edn. People's Medical Publishing House, Beijing.

Zhang ZB, Yu LJ, Yang KJ, Xu LW, et al. (2011). Association between single nucleotide polymorphisms (SNPs) at the promoter of adiponectin gene and essential hypertension in Chinese Korean and Han of Yanbian region. Yi Chuan 33: 54-59. 